\title{
Acoustic Assembly of Cell Spheroids in Disposable Capillaries
}

Yue $\mathrm{Wu}^{1}$, Zheng $\mathrm{Ao}^{1}$, Bin Chen ${ }^{1,2}$, Maram Muhsen ${ }^{1}$, Maria Bondesson ${ }^{1}$, Xiongbin Lu ${ }^{3}$ and Feng Guo ${ }^{1 *}$

1.Department of Intelligent Systems Engineering, Indiana University, Bloomington, Indiana 47405, United States

2.Department of Laboratory Medicine, Guangzhou First People's Hospital, Guangzhou Medical University, Guangzhou 510180, China.

3.Department of Medical and Molecular Genetics and Melvin and Bren Simon Cancer Center, Indiana University School of Medicine, Indianapolis, IN 46202, USA

*E-mail: fengguo@iu.edu

\begin{abstract}
:
Multicellular spheroids represent a promising approach to mimic 3D tissues in vivo for emerging applications in regenerative medicine, therapeutic screening, and drug discovery. Conventional spheroid fabrication methods, such as the hanging drop method, suffer from low throughput, long time, complicated procedure, and high heterogeneity in spheroid size. In this work, we report a simple yet reliable acoustic method to rapidly assemble cell spheroids in capillaries in a replicable and scalable manner. Briefly, by introducing a coupled standing surface acoustic wave, we are able to generate a linear pressure node array with 300 trapping nodes simultaneously. This enables us to continuously fabricate spheroids in a highthroughput manner with minimal variability in spheroid size. In a proof of concept application, we fabricated cell spheroids of mouse embryonic carcinoma (P19) cells, which grew well and retaineddifferentiation potential in vitro. Based on the advantages of the non-invasive, contactless and label-free acoustic cell manipulation, our method employs the coupling strategy to assemble cells in capillaries, and further advances 3D spheroid assembly technology in and easy, cost-efficient, consistent, and high throughput manner. This method could further be adapted into a novel 3D biofabrication approach to replicate compilated tissues and organs for a wide set of biomedical applications.
\end{abstract}

This is the author's manuscript of the article published in final edited form as: 


\section{Introduction}

In vivo, cells are constantly interacting with the extracellular matrix and/or directly physically contacting and communicating with surrounding cells to regulate complex biological functions like development, homeostasis, and disease progression. Currently, most studies in biomedical sciences are performed using 2D adherent cell culture methods, which provides a well-controlled, homogenous cell culture environment for most cell types. However, experimental data produced by $2 \mathrm{D}$ adherent cell culture cannot be completely translated into animal studies or clinical trials because they fail to recapitulate complicated signals among cells and 3D structure of cells growing in vivo [1] [2]. Multicellular spheroidal cultures address this challenge and represent better in vivo physiological conditions [3]. The spheroidal cell culture also better reflects 3D distributions of nutrients, metabolites, catabolites, and oxygen in vivo. Because of these features, spheroidal cell culture attracts significant attention from the fields of regenerative medicine, translational medicine, personalized medicine and highthroughput drug screening [5] [6] [7]. For applications of cancer treatment, tumor spheroids, compared to 2D culture, exhibit better histological and physiological features mimicking those of actual solid tumors and provide an advantageous model to investigate therapeutic interventions [8]. For applications in stem cell biology, spheroidal cell culture generally enhances the differentiation capability of stem and progenitor cells. For example, progenitor cells derived from salivary glands are able to differentiate into hepatocytic and pancreatic lineages only in 3D spheroidal culture but not in 2D adherent culture [9]. Moreover, organoids (intestinal, kidney, cerebral and optic cup) [10] [11] [12], cell spheroids derived from stem cells with a complex organ architecture, are obtained by in vitro 3D spheroid formation, but not by 2D culture. However, current spheroidal culture techniques, such as the hanging drop method, are laborious and do not fully meet the tremendous needs of replicability, scalability, and consistency of spheroid formation for these emerging biomedical applications.

To date, many engineering efforts have been made to fabricate spheroids with identical structure, morphology, physiology, and sufficient quantity. Several commercially available methods including hanging drop [13], spinner culture [14] [15], rotating wall vessels [16], nonattachable surface [17] are time-consuming, low-throughput, yield a poor spheroid uniformity and/or require a large number of cells. Taking the classical hanging drop for example, this method uses the gravity to slowly promote cell-cell interaction between suspended cells in 
hanging drops for reliable cell spheroid formation, while this method normally takes 7 days to form only 96 spheroids in a 96-well plate [13]. Other existing methods including microfluidics [18] [19] [20] [21], micromold [22], di-electrophoresis [23], and magnetic-assisted assembly [24] [25] require dedicated conditions such as complicated design and fabrication, medium modification, and/or cell labelling. Recently, acoustics became a promising alternative approach for spheroid fabrication because it provides excellent biocompatibility, flexibility, and contactless and label-free manipulation of cells while preserving the cells' native state [26] [27] [28]. The bulk acoustic wave resonator has been used to levitate cells into multiple paralleled layers vertically for the fabrication of cell spheroids [29] [30]. The acoustic streaming has been employed to aggregate cells in 24-well plate for the formation of cell spheroids [31]. However, it is challenging to reproducibly generate uniform spheroids in a high-throughput manner due to the complicated setup or the requirement of a sensitive temperature control. Recently, we have developed 3D acoustic tweezers for the 3D manipulation of single cells by introducing standing surface acoustic wave into microfluidics [32] and demonstrated the formation of cancer cell spheroids at 100 cells per hour with our SSAW microfluidic device [33]. However, complicated design and fabrication of microfluidic devices are still required, and the devices are relatively expensive mainly due to the price of the SSAW generator. Therefore, in order to develop a technology which would be widely applicable for acoustic cell spheroid fabrication in a standard cell biology laboratory, hospital, or pharmaceutical industry, an advanced spheroidal culture technique is highly desirable to achieve simpler setup, lower cost, higher throughput, and better uniformity. We here describe the generation of such a device and demonstrate its efficiency by assembling P19 cells into rapidly growing spheroids.

In this work, we demonstrated an acoustic assembly technique that can rapidly and highthroughput fabricate cell spheroids in commercially available capillaries. By introducing the standing surface acoustic wave into the disposable capillary with the oil-coupling strategy, a linear pressure node array with around 300 trapping nodes was generated. Thus, we could repeatedly form 300 spheroids of similar size and compositions in a capillary in less than 1 second and obtain around 100 stable spheroids in Petri dishes without cell dissociation in 30 minutes. Compared with traditional hanging drop method (96 spheroids per 7 days) [13], our acoustic cell assembly method (100 spheroids per day) could significantly enhance the efficiency for spheroid fabrication. Our method also enabled cardiomyocyte differentiation of mouse embryonic carcinoma cells. Moreover, the oil-coupling strategy allowed for re-usage 
of the relatively expensive SSAW generator and simultaneous processing of multiple disposable capillaries to further enhance the throughput. With its simplicity, rapid spheroid formation, high-throughput, and non-invasiveness, the acoustic cell assembly approach presented here can be highly applicable for many 3D biofabrication applications.

\section{Working mechanism}

We developed a simple method to rapidly assemble cell spheroids in a capillary using standing surface acoustic wave (SSAW). The working mechanism of acoustic assembly of cell spheroids is illustrated in Figure 1. Our acoustic assembly device included disposable capillaries and a reusable SSAW generator, which could be coupled together with a thin layer of coupling gel. The reusable SSAW generator was built by depositing a pair of interdigital transducers (IDTs) on a piezoelectric substrate $\left(\mathrm{LiNbO}_{3}\right)$. To produce a linear cell assembly array, we chose a round capillary with an inside diameter of around the half wavelength of the SSAW and coupled the capillary in the SSAW-activated region of the substrate along the propagation direction of SSAW. Cell suspensions were introduced into the capillary by capillary force. Once the radio frequency signal was applied to the IDT pair of the SSAW generator, a SSAW was generated and propagated on the piezoelectric substrate and leaked into the glass capillary through a thin layer of coupling oil between the SSAW generator and the capillary. The SSAW locally introduced into the capillary formed a periodic distributed acoustic field in the capillary after interacting with and reflecting by the capillary wall. Due to the gradient of the acoustic field, an acoustic radiation force was generated, which pushed suspended cells to the pressure nodes or pressure antinodes. In a standing acoustic wave field, the primary acoustic force $\left(F_{r}\right)$ on a particle or a cell [34] [35] can be expressed as [36]

$$
\begin{gathered}
F_{r}=-\left(\frac{\pi p_{0}{ }^{2} V_{p} \beta_{m}}{2 \lambda}\right) \emptyset(\beta, \rho) \sin (2 \mathrm{kx}) \\
\emptyset=\frac{5 \rho_{p}-2 \rho_{m}}{2 \rho_{p}+\rho_{m}}-\frac{\beta_{p}}{\beta_{m}}
\end{gathered}
$$

where $p_{0}, V_{p}, \lambda, k, \mathrm{x}, \rho_{m}, \rho_{p}, \beta_{m}$, and $\beta_{p}$ correspond to pressure amplitude, particle volume, acoustic wavelength, wave vector, distance from a pressure node, density of the medium, density of particles, compressibility of the medium, and compressibility of particles, respectively. The acoustic contrast factor $(\phi)$ determines whether the particle or cell moves to the pressure nodes or pressure antinodes, and usually, the cell suspended in normal culture medium will move towards the pressure nodes because $\phi$ is positive. The distance between 
neighboring nodes (the distance between the center of two cell clusters) is always the wavelength of SSAW on the substrate. By tuning the cell concentration, suspended cells were moved and aggregated into a linear cell assembly array. After incubation in the capillary, the cells within an assembly formed stable cell-cell contacts to maintain cell assembly. Next, the assembled cells were transferred into ultralow attachment dishes, where they were kept growing as cell spheroids for long-term culture. To induce differentiation of P19 cells, the cell spheroids were transferred to adherent culture dishes, and differentiation markers were assessed.

\section{Experiments}

The SSAW generator was fabricated by a standard soft lithography and lift-off process [37]. A 7- $\mu$ m-thick photoresist layer (S1813, MicroChem, MA) was spin-coated on the piezoelectric substrate (a 500- $\mu$ m-thick, double-side polished, $128^{\circ} \mathrm{YX}$-propagation $\mathrm{LiNbO}_{3}$ wafer). Then, the designed IDT patterns of 40 electrode pairs with the $75 \mu \mathrm{m}$ finger width and periodic spacing were transferred from the plastic mask (Kunshan Kaisheng Electronics Co., Ltd, China) to the substrate by UV exposure. The IDT patterns were developed in a photoresist developer (MF CD-26, Microposit, MA) and deposited with double metal layers $(\mathrm{Cr} / 50 \AA$, Au/600 Å) by a thermal evaporation (JSD-350, Anhui Jiaoshuo Vacuum Technology Co., Ltd, China). IDTs on the piezoelectric substrate were finally obtained after a standard lift-off process. The resonant frequency of the fabricated SSAW generator was measured as around 20.7 MHz using a network analyzer (E8362C, Agilent, CA).

Mouse embryonic carcinoma cells (P19, ATCC, VA) were cultured in Alpha Modified Eagle's Medium (Corning, NY) supplemented with 10\% fetal bovine serum (Sigma-Aldrich, MO), 100 $\mathrm{U} / \mathrm{mL}$ penicillin and $100 \mu \mathrm{g} / \mathrm{mL}$ streptomycin (Invitrogen, PA), in a humidified incubator at $5 \%$ $\mathrm{CO}_{2}$ and $37^{\circ} \mathrm{C}$. For the experiments, cells were suspended by dissociation of cells with $0.25 \%$ trypsin-EDTA (Invitrogen, PA), followed by centrifugation of dissociated cells at $800 \mathrm{rpm}$ for 5 min at room temperature, and re-suspension in culture medium at a final concentration of 5 to 10 million cells per $\mathrm{mL}$. Cells were observed using microscopy (IX83, Olympus, Japan) and counted using a hemocytometer.

P19 cells were acoustically-assembled into cell clusters in capillaries. After coating a thin layer of oil (Sigma, MO) to the SSAW generator, a glass capillary (inside diameter $=200 \mu \mathrm{m}$; outside 
diameter $=300 \mu \mathrm{m}$; length $=5 \mathrm{~cm}$; VitroCom, NJ) sterilized by UV exposure was used to load the P19 cell suspension and was coupled to the SSAW generator through the thin oil layer. P19 cells were aggregated into a linear array in the capillary by energizing the IDT pair with a radio frequency signal generated by a function generator (AFG3102C, Tektronix, CA) and amplified by an amplifier (25A100A, Amplifier Research, CA). The movement of cells was monitored and recorded by microscope (IX83, Olympus, Japan) equipped with a CMOS camera (ORCA-Flash 4.0, HAMAMATSU, Japan) connected to a computer. The P19 cells were acoustically-aggregated within 1 minute by an input acoustic power from 2 to $10 \mathrm{Vpp}$. Then cell clusters were incubated in the capillary for about 30 minutes to allow the suspended cells to form stable of cell-cell contacts. The cell images were analyzed using ImageJ 1.46 software.

To form cell spheroids, assembled P19 cell were transferred from the capillary to an ultra-low attachment petri dish or well plate (Corning, PA) with fresh cell culture medium. The petri dish or well plate with cell clusters was incubated in an in-situ chamber (Tokai Hit, Japan) integrated on the microscope and maintained at $5 \% \mathrm{CO}_{2}$ and $37^{\circ} \mathrm{C}$. The cell clusters were imaged and recorded daily from day 0 to day 3. After 3-days of culture in ultra-low attachment 24-well plates (Corning, PA), the P19 cell spheroids were transferred to adherent 24-well plates (Corning, PA) for cardiomyocyte differentiation. Cell spheroids were allowed to adhere to the 24-well plate overnight following treatment with complete DMEM medium containing $1 \%$ DMSO (Sigma-Aldrich, MO) for 7 days. After the DMSO-induced cardiomyocyte differentiation, cells were fixed with $4 \%$ formalin (Affymetrix, CA) and permeabilized with icecold methanol (Sigma, MO). Cells were stained with anti-Troponin I antibody (Abcam, CA) followed by secondary antibody, goat anti-rabbit Alexa 594 (Thermo Fisher, IN). Cells were counterstained with DAPI (Thermo Fisher, IN) and imaged using a microscope (IX83, Olympus, Japan) using a 40X objective. Meanwhile, differentiated P19 cells were harvested for RNA extraction (Qiagen, MD). Troponin I expression was analyzed using one step qRTPCR kit (Thermo Fisher, IN). Fold change was calculated as $2 \Delta \Delta \mathrm{Ct}$ based on expression fold change against P19 parental cell line and normalized against GAPDH housekeeping gene.

In order to completely record the cell spheroid development process, a group of P19 cell spheroids were cultured for one week and cellular viability was monitored every $24 \mathrm{~h}$. Living cells were labelled with green fluorescent using calcein AM, and dead cells were labelled with 
red fluorescent using ethidium homodimer-1 (live/dead viability kit, L3224, Thermo Fisher Scientific Inc.). The cellular viability was recorded by calculating the ratio of green fluorescent area sizes over total spheroid. To measure P19 spheroid proliferation, cell number was measured by CCK-8 kit (Sigma, USA) every 24 hour. Cell spheroids were harvested from nonadherent plate and plated into 96-well plate. The spheroids were co-incubated with CCK-8 agent for 4 hours at $37^{\circ} \mathrm{C}$ incubator. The plate was then read at $450 \mathrm{~nm}$ for absorbance. Cell numbers were calculated based on stand curve plotted using serial titration of P19 parental cell line.

\section{Results and discussion}

The assembled device generated a non-uniform pressure distribution in the capillary by introducing leaked SSAW from the SSAW generator to the capillary with a thin layer of coupling oil. Next, the cells were moved and aggregated to a linear pressure node array in the capillary (Fig. 2A). In our design, the length of the cell assembly region was about $4.5 \mathrm{~cm}$; the distance between two adjacent pressure nodes was $150 \mu \mathrm{m}$ (as the half wavelength); and the length, inside and outside diameter of the glass capillary was $5 \mathrm{~cm}, 200 \mu \mathrm{m}$, and $300 \mu \mathrm{m}$, respectively. Therefore, the number of pressure nodes (cell clusters) created in the capillary was approximately 300 , offering a reasonable throughput. Fig. 2B shows the time-lapse images of P19 cells moving into the pressure nodes in the capillary. When the acoustic field was applied, the cells within one fourth wavelength distance from the center of the nearest pressure node aggregated to form cell clusters within 1 second (See ESI Video 1). The cells became well assembled into a linear cell cluster array in the capillary up to the region of IDTs and the gap between the IDT pair.

The non-invasiveness is one of the biggest advantages of our acoustic cell assembly technique. We measured the viability of P19 cells before and after acoustic aggregation by live/ dead cell staining. The P19 cells were simultaneous fluorescently stained using calceinAM and ethidium homodimer-1, which is indicative of viable and dead cells, respectively. The control group without any treatment and the experimental group with acoustic assembly treatment were repeated three times, respectively. As shown in Fig. $2 \mathrm{C}$, there was no significant difference in viability of $\mathrm{P} 19$ cells without or with the acoustic treatment $(87.1 \pm 0.87 \%$ 
vs. $84 \pm 3.7 \%$, respectively, $\mathrm{P}=0.284$ ). Thus, we demonstrated that our acoustic cell assembly had a good biocompatibility.

We previously described the mechanism of acoustic assembly of cell spheroids and aggregation of P19 cells in a capillary. To extend this technique to broader applications in the biological laboratory or pharmaceutical industry, we developed a technique compatible with commercially available and widely used cell culture approaches and consumables, such as Petri dishes and well-plates. In this regard, we further extended our method for capillary-free, long-term culture of cell spheroids. After acoustically-assembly of P19 cells into clusters in the capillary, strong cell-cell connections were formed during the in vitro room temperature incubation (10-30 minutes), allowing for stable cell clusters to be transferred from the capillary to an ultralow attachment dish. We found that 30 minutes is the optimal incubation time for P19 cell assembly as determined by testing different incubation times (10, 20, and 30 minutes).

After forming stable cell contacts, we carefully flushed out the cell assemblies from the capillary to an ultralow attachment dish filled with cell culture medium. Compared to microfluidic approaches, our method employed commercially available capillaries, avoiding the complicated fabrication of microfluidic chambers as well as the dead volume between the tubing and microfluidic inlets or outlets. The recovery rate of the cell assembly is critical for the cell spheroid culture and subsequent cardiomyocyte differentiation studies. Here, we collected around 100 stable cell clusters in a petri dish from a capillary with around 300 cell assemblies. The cell-to-cell boundary became blurred as the cells formed spheroids during the 12-hour-culture after the transfer of the cell clusters into well plates. The growth of cell spheroids in the ultralow attachment dish was monitored and recorded by time-lapse microscopy (Fig. 3). After 3 days of culture, the average size of the cell spheroids increased from $139 \mu \mathrm{m}$ to $292 \mu \mathrm{m}$, which is ready for the differentiation experiments.

To further demonstrate the developed P19 cell spheroids, acoustically-assembled P19 cell spheroids were cultured for 7 days and characterized with the viability and prefiltration tests. For day 1 and day 2, the average size of cell spheroids was less than $200 \mu \mathrm{m}$ and there was almost no cell death (Fig. 4A). The P19 cell spheroids grow gradually to the average diameter around $600 \mu \mathrm{m}$, and showed a significant stratification until day 7. The calceim AM staining of 
developed P19 spheroid at day 7 (in green) indicated the proliferative zone, while the homodimer-1 staining of developed P19 spheroid at day 7 (in red) showed the necrotic zone. To some extent, spheroids at this state displayed inherent metabolic (oxygen, carbon dioxide, nutrients, and wastes) gradients similarly to poorly vascularized tumors (Fig. 4A). In addition, CCK-8 kit was used to measure the P19 spheroid expansion rate. The cell number of the developed P19 cell spheroids was increasing dramatically during the 7-day culture to show a good proliferation (Fig. 4B). Thus, we demonstrated a method to form intact and viable cell spheroids.

To demonstrate that the assembled P19 spheroids preserve the biological traits of the natural embryonic body, we analysed the differentiation capacity of acoustically-assembled P19 spheroids. P19 cell spheroids can differentiate into cardiomyocytes and skeletal muscle cells upon 1\% DMSO induction and adherent conditions [38]. To validate that the acoustically assembled P19 spheroids had the same traits, P19 cells were first assembled by our device as described above and were allowed to form spheroids of around $300 \mathrm{~mm}$ diameter by culturing them for 3 days in ultralow attachment plates. After the spheroids reached the desired size, they were transferred onto an adherent plate for attachment (Fig. 5A). Spheroids were allowed to adhere to the plate for overnight and were then treated with $1 \%$ DMSO. After induction for 7 days, we analysed cardiomyocyte marker expression by immunofluorescence. As shown in Fig.5B, assembled P19 spheroids were able to develop into cardiomyocytes, as indicated by cardiomyocyte marker -Troponin I. In contrast, without DMSO or acoustic assembly, such differentiation was not observed. This proved that our acoustic assembly method did not alter the differentiation potential of P19 embryonic cells. In addition, Troponin I expression of differentiated P19 spheroids was also analyzed by qRT-PCR. As shown in Fig. 5C, DMSO induced a $\sim 4$ folds (10,000 times) increase in the expression of Troponin I gene in P19 spheroids. This result further confirmed the cardiomyocyte differentiation of P19 spheroids induced by DMSO, which demonstrated that the developed P19 cell spheroids always maintained differentiation potential. Thus, our acoustic assembly method allows for a much easier and higher-throughput method to generate embryonic bodies for research applications.

\section{Conclusion}


By coupling disposable capillaries to a reusable SSAW generator, a large number of pressure nodes can be produced to aggregate cells for the formation of cell spheroids. This acoustic cell assembly method enables rapid, noninvasive and high throughput means to fabricate cell spheroids (aggregation of cell within 1 second; almost no impact on the cell viability; generation of 300 cell clusters in one capillary). Moreover, the coupling strategy combines a simple operation with low cost, no cross-contamination, and high-throughput parallel assembly of multiple capillaries. These advantages make the acoustic cell assembly technique presented here promising for biofabrication of cell spheroids, organoids, and other spheroidbased 3D architectures.

\section{Acknowledgments}

This project was supported by the departmental start-up fund of Indiana University Bloomington and the Vice Provost for the Research through the Faculty Research Support Program (Indiana University Bloomington).

\section{ORCID iDs}

Feng Guo https://orcid.org/0000-0001-9103-3235 


\section{References}

[1] Pampaloni F, Reynaud E G and Stelzer E H K 2007 The third dimension bridges the gap between cell culture and live tissue Nature Reviews Molecular Cell Biology 8839

[2] Baraniak P R and McDevitt T C 2012 Scaffold-free culture of mesenchymal stem cell spheroids in suspension preserves multilineage potential Cell Tissue Res 347 $701-11$

[3] Fennema E, Rivron N, Rouwkema J, van Blitterswijk C and de Boer J 2013 Spheroid culture as a tool for creating 3D complex tissues Trends Biotechno/31 108-15

[4] Sutherland R 1988 Cell and environment interactions in tumor microregions: the multicell spheroid model Science 240 177-84

[5] Mueller-Klieser W 1987 Multicellular Spheroids. A review on cellular aggregates in cancer research vol 113

[6] Mueller-Klieser W 1997 Three-dimensional cell cultures: from molecular mechanisms to clinical applications American Journal of Physiology-Cell Physiology 273 C1109-C23

[7] Sasai Y 2013 Next-Generation Regenerative Medicine: Organogenesis from Stem Cells in 3D Culture Cell Stem Cel/ 12 520-30

[8] Dertinger H and Lücke-Huhle C 1975 A Comparative Study of Post-irradiation Growth Kinetics of Spheroids and Monolayers International Journal of Radiation Biology and Related Studies in Physics, Chemistry and Medicine 28 255-65

[9] Okumura K, Nakamura K, Hisatomi Y, Nagano K, Tanaka Y, Terada K, Sugiyama T, Umeyama K, Matsumoto K, Yamamoto T and Endo F 2003 Salivary gland progenitor cells induced by duct ligation differentiate into hepatic and pancreatic lineages Hepatology 38 104-13

[10] Laschke M W and Menger M D 2017 Life is 3D: Boosting Spheroid Function for Tissue Engineering Trends Biotechno/35 133-44

[11] Clevers H 2016 Modeling Development and Disease with Organoids Cell 165 1586-97

[12] Huch M and Koo B K 2015 Modeling mouse and human development using organoid cultures Development 142 3113-25

[13] Timmins N, Dietmair S and Nielsen L 2004 Hanging-drop multicellular spheroids as a model of tumour angiogenesis Angiogenesis 7 97-103 
[14] Lei Y, Jeong D, Xiao J and Schaffer D V 2014 Developing Defined and Scalable 3D Culture Systems for Culturing Human Pluripotent Stem Cells at High Densities Cellular and Molecular Bioengineering 7 172-83

[15] Laschke M W and Menger M D 2017 Life is 3D: Boosting Spheroid Function for Tissue Engineering Trends Biotechno/35 133-44

[16] Lei Y and Schaffer D V 2013 A fully defined and scalable 3D culture system for human pluripotent stem cell expansion and differentiation Proceedings of the National Academy of Sciences 110 E5039-E48

[17] Napolitano A P, Chai P, Dean D M and Morgan J R 2007 Dynamics of the SelfAssembly of Complex Cellular Aggregates on Micromolded Nonadhesive Hydrogels Tissue Engineering 13 2087-94

[18] Patra B, Chen Y H, Peng C C, Lin S C, Lee C H and Tung Y C 2013 A microfluidic device for uniform-sized cell spheroids formation, culture, harvesting and flow cytometry analysis Biomicrofluidics 754114

[19] Hsiao A Y, Torisawa Y-s, Tung Y-C, Sud S, Taichman R S, Pienta K J and Takayama S 2009 Microfluidic system for formation of PC-3 prostate cancer co-culture spheroids Biomaterials 30 3020-7

[20] Chan H F, Zhang Y, Ho Y P, Chiu Y L, Jung Y and Leong K W 2013 Rapid formation of multicellular spheroids in double-emulsion droplets with controllable microenvironment Sci Rep 33462

[21] Torisawa Y-s, Chueh B-h, Huh D, Ramamurthy P, Roth $T$ M, Barald K F and Takayama S 2007 Efficient formation of uniform-sized embryoid bodies using a compartmentalized microchannel device Lab Chip 7 770-6

[22] A. S A, Nalin T, Y. S J, B. S V and R. M J 2014 Micro-Mold Design Controls the 3D Morphological Evolution of Self-Assembling Multicellular Microtissues Tissue Engineering Part A 20 1134-44

[23] Albrecht D R, Underhill G H, Wassermann T B, Sah R L and Bhatia S N 2006 Probing the role of the multicellular organization in three-dimensional microenvironments Nature Methods 3369

[24] Souza G R, Molina J R, Raphael R M, Ozawa M G, Stark D J, Levin C S, Bronk L F, Ananta J S, Mandelin J, Georgescu M-M, Bankson J A, Gelovani J G, Killian T C, Arap W and Pasqualini R 2010 Three-dimensional tissue culture based on magnetic cell levitation Nature Nanotechnology 5291 
[25] Alessandro T, Gozde D N, Kaushik S, Vigneshwaran M, Bukre C, Rami E A and Utkan D 2018 Magnetically Guided Self-Assembly and Coding of 3D Living Architectures Adv Mater 301705034

[26] Friend J and Yeo L Y 2011 Microscale acoustofluidics: Microfluidics driven via acoustics and ultrasonics Reviews of Modern Physics 83 647-704

[27] Ding X, Li P, Lin S-C S, Stratton Z S, Nama N, Guo F, Slotcavage D, Mao X, Shi J, Costanzo F and Huang T J 2013 Surface acoustic wave microfluidics Lab Chip 13 $3626-49$

[28] Laurell T, Petersson F and Nilsson A 2007 Chip integrated strategies for acoustic separation and manipulation of cells and particles Chemical Society Reviews 36 492-506

[29] Bazou D, Kearney R, Mansergh F, Bourdon C, Farrar J and Wride M 2011 Gene Expression Analysis of Mouse Embryonic Stem Cells Following Levitation in an Ultrasound Standing Wave Trap U/trasound in Medicine \& Biology 37 321-30

[30] Jian L, A. K L, O. E G, Jinsheng X, Mingwen M, M. P W, K. J S and Terence C W 2007 Functional three-dimensional HepG2 aggregate cultures generated from an ultrasound trap: Comparison with HepG2 spheroids Journal of Cellular Biochemistry 102 1180-9

[31] Kurashina Y, Takemura K and Friend J 2017 Cell agglomeration in the wells of a 24-well plate using acoustic streaming Lab Chip 17 876-86

[32] Guo F, Mao Z M, Chen Y C, Xie Z W, Lata J P, Li P, Ren L Q, Liu J Y, Yang J, Dao M, Suresh S and Huang T J 2016 Three-dimensional manipulation of single cells using surface acoustic waves $P$ Natl Acad Sci USA 113 1522-7

[33] Chen K J, Wu M X, Guo F, Li P, Chan C Y, Mao Z M, Li S X, Ren L Q, Zhang R and Huang T J 2016 Rapid formation of size-controllable multicellular spheroids via 3D acoustic tweezers Lab Chip 16 2636-43

[34] Bruus H 2012 Acoustofluidics 7: The acoustic radiation force on small particles Lab Chip 12 1014-21

[35] Bruus H 2012 Acoustofluidics 2: Perturbation theory and ultrasound resonance modes Lab Chip 12 20-8

[36] L. P. Gorkov, Soviet Physics - Doklady, 1962, 6, 773-775

[37] Guo F, Li P, French J B, Mao Z, Zhao H, Li S, Nama N, Fick J R, Benkovic S J and Huang T J 2015 Controlling cell-cell interactions using surface acoustic waves 
Proceedings of the National Academy of Sciences 112 43-8

[38] Skerjanc I S 1999 Cardiac and Skeletal Muscle Development in P19 Embryonal Carcinoma Cells Trends in Cardiovascular Medicine 9 139-43 


\section{Figures and captions}

Figure 1: Schematics of the acoustic assembly of cell spheroids. The acoustic cell assembly device was made by coupling disposable capillaries onto a reusable standing surface acoustic wave (SSAW) generator with a pair of interdigital transducers (IDTs). Suspended P19 cells were aggregated into cell clusters and incubated in the capillary $(A)$, transferred and cultured in a non-attachable dish to form cell spheroids (B), differentiated into cardiomyocytes after culturing in an attachable dish (C).

Figure 2: Acoustic cell aggregation in the capillary. (A) Mechanism of acoustic cell assembly in a capillary. The waves indicate the pressure distribution in the microchannel. The arrows on the cells indicate the direction of cell movement. (B) Experimental time-lapse images of cells clustering into a linear assembly array after applying the acoustic field within 1 second. (C) The viability of P19 cells before (OFF) or after (ON) the acoustic cell aggregation was $87.1 \pm$ $0.87 \%$ or $84 \pm 3.7 \%$, respectively. Scale bar: $200 \mu \mathrm{m}$

Figure 3: P19 Cell spheroid formation and culture. (A) P19 cell clusters were transferred onto an ultralow attachment dish and cultured for 3 days. Acoustically-assembled cell clusters grew into cell spheroids while other randomly distributed cells were degraded after one- or two-days culture. (B) The sizes of P19 cell clusters and spheroids were measured from day 0 to day 3 until spheroids were transferred to adhesive cell culture dishes for differentiation studies. Scale bar: $200 \mu \mathrm{m}$

Figure 4: Long-term culture of P19 Cell spheroids. (A) P19 cell spheroids were cultured in an ultralow attachment dish for one week. Live cells were labelled with calcein AM (green), and dead cells were labelled with Ethidium homodimer-1(red). (B) Proliferation test of P19 cell spheroids with CCK-8 kit (Sigma, USA). Scale bar: $200 \mu \mathrm{m}$.

Figure 5: P19 cell spheroid adhesion and differentiation. (A) After being transferred to adherent petri dishes, P19 cell spheroids adhered to the substrate during the culture from day 3 to day 4. (B) The assembled P19 spheroids treated with 1\% DMSO were able to develop into cardiomyocyte, as indicated by the cardiomyocyte marker Troponin I (indicated by red fluorescence). In contrast, without DMSO or acoustic assembly, such differentiation was not observed. (C) Troponin I expression of P19 cell spheroids with DMSO was about 4 folds (10,000 times) higher than that of P19 cell spheroids without DMSO using one step qRT-PCR kit after 7 days DMSO treatment. Scale bar: $100 \mu \mathrm{m}$ 